\title{
Histological and Histochemical Studies on the Nervous Influence on Minced Muscle Regeneration of Triceps Surae of the Rat ${ }^{1,2}$
}

\author{
FRANZ S. F. MONG 3,4 \\ Department of Anatomy, University of Michigan, Medical School, Ann Arbor, \\ Michigan 48104
}

\begin{abstract}
The degree of minced rat muscle regeneration in the absence of nerve fibers was compared with that of normal regenerates between one and 270 days postoperatively. Up to around 30 days, the number of muscle fibers and their morphology were comparable in both normal innervated and denervated regenerates; both showed clear cross striations and peripherally located nuclei. Histochemically, SDH and myofibrillar ATPase $(\mathrm{pH}=9.4)$ reactions were positive, but there were no typical signs of fiber types in either case of regeneration. The only consistent difference in the early period was the smaller fiber cross sectional areas in denervated regenerates than in innervated ones. Starting about 40 days, the muscle fibers in innervated regenerates became differentiated into different fiber types (fast-twitch-oxidative-glycolytic, FOG., fast-twitch-glycolytic, FG., slow-twitch-oxidative, $S O$.) but there were no such activities in denervated regenerates, although their SDH and myofibrillar ATPase reactions remained positive for a long time. Degenerating muscle fibers could no longer be identified in innervated regenerates. In the denervated regenerates, however, muscle fibers underwent atrophic or degenerative changes and were replaced by connective tissue. The complete disappearance of muscle fibers varied with individual regenerates. In some cases, it occurred about 90 days and in others, traces of muscle fibers could still be seen as late as $\mathbf{1 5 0}$ days postoperatively. Thus, nerves seem to be important primarily in the late phase of regeneration; namely, differentiation of fiber types and maintenance of the structural integrity of muscle fibers.
\end{abstract}

That muscle can regenerate after trauma and disease has been known for more than a century, but the problem has been intensively studied only during the last two or three decades (Hudgson and Field, '73, for review). The most traumatic injury used to cause muscle to regenerate is mincing (Studitsky et al., '63; Carlson, '68, '70) in which one removes a muscle (triceps surae [gastrocnemius, soleus, and plantaris] in the present study), minces it into fragments of one $\mathrm{mm}^{3}$ in size and replaces them in the original muscle bed. The implanted fragments first degenerate and, from these degenerating muscles, myogenic elements (myoblasts) emerge. These myoblasts then fuse to form multinucleated structures called myotubes which eventually differen- tiate into mature muscle fibers. Carlson and Gutmann ('72) found that the mature regenerates proved to be functional upon direct stimulation and behaved like fast contracting muscle; however, histochemical studies of specific muscle fiber types were not made.

One question frequently asked in the study of muscle regeneration is whether nerves are necessary for muscles to regenerate. Studitsky et al. ('63) first stated that

\footnotetext{
'Supported in part by NIH predoctoral training grant and a grant from Muscular Dystrophy Association of America to Dr. B. M. Carlson.

${ }^{2}$ Reported earlier in abstract form (Mong, 75 ).

${ }^{3}$ This material was taken in part from a thesis for the degree of Doctor of Philosophy, University of Michigan.

${ }_{4}^{4}$ Present address: Department of Anatomy, Medical College of Virginia, Virginia Commonwealth University, Richmond, Virginia 23298.
} 
fusion of myoblasts to form myotubes required the presence of nerves and that denervation would stop muscle regeneration. Zheneskaya ('62) maintained that, in the absence of nerves, myoblasts and myotubes developed intensively. Only further differentiation of muscle fibers was retarded. Both of these studies involved mincing procedures. In the post-ischemic muscle regeneration of rabbits, however, Allbrook and Aitkin ('51) found few histological differences between normal and denervated regenerates. Hsu ('71) studied minced muscle regeneration of frogs and observed, in the denervated regenerates, muscle fibers with cross-striation and peripheral nuclei. These muscle fibers, however, eventually degenerated. Neither in the rabbit (Allbrook and Aitkin, '51) nor in the frog (Hsu, 71) was the degree of muscle fiber maturity determined in terms of histochemical fiber types. Thus, the purpose of the present study is two-fold: (1) to determine whether and when distinct fiber types would return in normal innervated muscle regenerates and (2) to investigate whether or not nerve is necessary for regeneration of minced muscle and, if so, the extent to which muscle fiber types, size (cross-sectional area), and morphology depend on the presence of nerve fibers.

\section{MATERIALS AND METHODS}

Male Sprague Dawley rats, 70-100 gm in weight, were operated upon according to the technique used by Studitsky et al. ('63) and Carlson ('68, '70). The experiment was divided into three series:

I. Normal control: Normal innervated muscle regeneration

II. Experimental: Denervated muscle regeneration

III. Quantitative comparison between normal and denervated muscle regeneration.

\section{Series I: Normal, innervated muscle regeneration}

In this series, all the nerves were left in situ. Attention was focused on (1) morphological development, (2) return of nerve fibers, (3) return of motor end plates (MEP's), and (4) muscle fiber type differentiation.

In all, 80 animals were used and the regenerates were examined from day 1 to day 270 after the operation. Of the 80 animals, 23 were used to study the return of nerve fibers, with the modified Palmgren's (Hsu, '71) silver stain method (9- $\mu$ m thick longitudinal paraffin sections), 14 were used to study the return of MEP's by Gomori's acetylthiocholinesterase method (Pearse, '72, 10- $\mu \mathrm{m}$ thick frozen section), and the rest (43 animals) were used to check the histochemical differentiation of muscle fiber types by the SDH reaction (Nachlas et al., '57) and the myofibrillar ATPase $(\mathrm{pH}=9.4)$ reaction of Padykula and Herman ('55). The classification of three fiber types: FOG (fast-twitch-oxidative-glycolytic), $F G$ (fast-twitch-glycolytic) and $S O$ (slow-twitch oxidative) is according to the terminology of Peter et al. ('72). Frozen sections $10-\mu \mathrm{m}$ thick were used for both reactions.

Control sections for the histochemical reactions were prepared in the same fashion except that the substrates (acetylthio-choline, sodium succinate, and ATP) for AChE, SDH, and myofibrillar ATPase respectively, were eliminated from the incubation media. Routine hematoxylin and eosin staining was used for morphological examinations in both cross and longitudinal sections.

\section{Series II: Denervated minced muscle regeneration}

The sciatic nerve of 43 animals was cut high in the gluteal region above its bifurcation before the actual muscle mincing. The nerve stumps distal to the cut were completely removed down to the level of the ankle joint. The nerve stump proximal to the cut was tied and placed between the skin and gluteal muscles to prevent nerve regrowth to the regenerates. Attention was focused on (1) morphological development and (2) histochemical fiber type differentiation. The staining methods used were the same as those used in Series I. Random 
longitudinal sections were stained with modified Palmgren's method to make certain that there were no nerve fibers in the denervated regenerates.

\section{Series III: Quantitative comparisons between normal and denervated muscle regeneration}

As degenerating muscle fibers and centrally located nuclei are encountered in both normal and denervated regenerates, quantitative studies were carried out to compare the two. The parameters are the following:

(1) Cross-sectional area of muscle fibers

(2) Total number of muscle fibers

(3) Percentage of muscle fibers with peripheral nuclei per 100 nucleated muscle fibers counted

(4) Percentage of normal muscle fibers (as opposed to degenerating muscle fibers) per 50 fibers counted.

Before paraffin embedding, the distal tendon was discarded and each regenerate was divided into three equal segments (proximal, middle, and distal). About thirty serial cross sections from the distal end of each segment were taken and mounted. The paraffin blocks were then trimmed so that longitudinal sections could be cut. All three segments of each regenerate were serially sectioned and the ribbons of each segment were divided into six equal portions. Thirty to thirty-five sections from adjacent portions of the ribbons were selected so that five slides could be made (No. 1 to No. 5). The purpose of this maneuver was to make certain that a random sample could be selected throughout the regenerate.

The cross-sectional areas of muscle fibers was measured by projecting the cross sections of regenerates under high power $(x$ 40) to a paper screen which contained a rectangular outline $(20 \times 25 \mathrm{~cm})$ in the center. The muscle fibers falling into this rectangle were traced individually along the contour of the fibers and measured with a planimeter. The fibers in one rectangle were considered as one sample. The mean cross-sectional area $\left(\mu \mathrm{m}^{2}\right)$ of each re- generate was eventually calculated from four samples.

For estimation of total muscle fiber numers, a grid $(10 \times 10,100$ divisions) was inserted into the eyepiece. Under high power $(\times 40)$, the grid was moved stepwise and successively throughout the entire cross section. Fibers included in the grid were counted and added together.

For the estimation of the percentage of muscle fibers with peripheral nuclei per 100 fibers counted, a line was set up to divide the field of the microscope into two halves. Under high power $(\times 40)$ the line swept across the entire section, passing through its center. Muscle fibers touched by the line were observed and those with nuclei were tabulated as central or peripheral until one sample (one sample $=100$ nucleated muscle fibers) was collected. In total, six samples were collected from each segment of the regenerated. For the estimation of the percentage of normal muscle fibers per 50 fibers counted, longitudinal sections were used and the line (in the eyepiece) swept through the distal end of the sections of each segment. Muscle fibers touched by the line were counted and tabulated as normal or degenerating mucle fibers until one sample (one sample $=50$ fibers) was collected. Again six samples were collected from each segment of the regenerate.

Statistical analyses (Three-Way Analysis of Variance: Winer, '71) were carried out on parameters (3) and (4). Regenerates of age $10,20,30$ and 40 days were tested and two normal and two denervated regenerates were used for each date.

\section{RESULTS}

\section{Series I: Normal, innervated muscle regeneration}

The time and events of normal, innervated minced muscle regeneration have been reported on in detail (Carlson, '68, 70 ).

The nerve fibers began sprouting from the nerve trunk into the regenerates about six or seven days after the operation. However, the nerves did not come in contact 
with the regenerating muscle fibers until a week later. Figure 1 shows nerve twigs spreading among muscle fibers of a 30-dayold regenerate. Motor end plates (MEP's) also returned to the regenerates. Starting from the middle of the second week, positive AChE could be seen in the periphery of the regenerates, but the reaction products were distributed in a diffuse manner along the regenerating muscle fibers. The
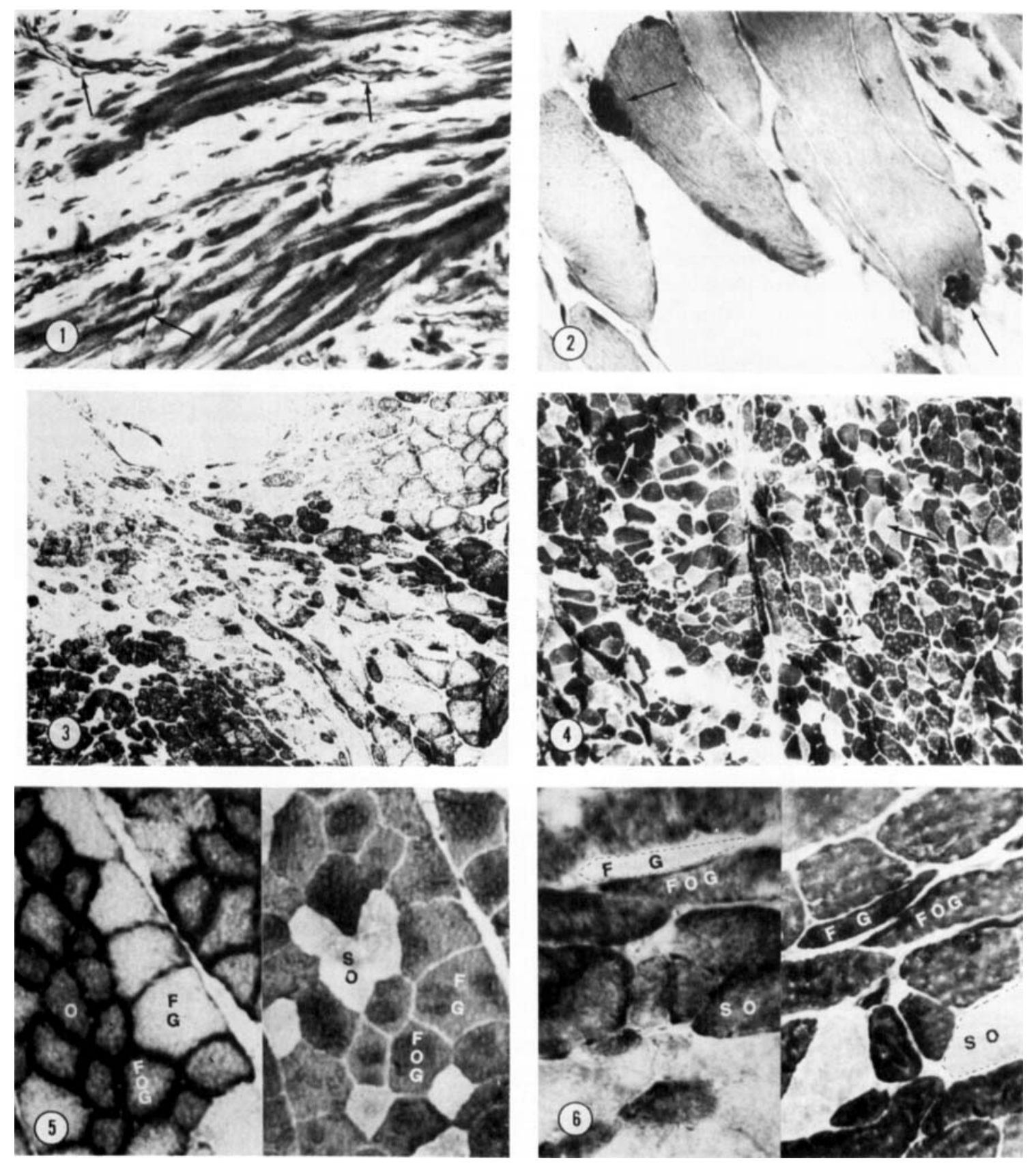
first sign of localized AChE reaction appeared around 20 days. Figure 2 shows the MEP's of a 45-day-old regenerate.

Fiber type differentiation also occurred in the normal regenerates. In the early stage (10-30 days), there was no sign of typical fiber type differentiation, although the reactions of SDH and myofibrillar ATPase were both positive. The first sign of typical fiber type differentiation occurred around 40 days. Light and dark fibers could be seen both in SDH and in myofibrillar ATPase reactions (figs. 3, 4). The three fiber types (FOG, FG and SO) of normal gastrocnemius and normal regenerates are shown in figures 5 and 6 , according to the terminology of Peter et al. ('72).

\section{Series II: Denervated minced muscle regeneration}

The time and events of the denervated regeneration were surprisingly similar to those of normal innervated regenerates. Myoblasts, myotubes and three zones of

Fig. 1 Thirty-day-old regenerate. Sections stained with Hsu's modified Palmgren silver method. It shows nerve fibers (arrows) spreading in among the regenerating muscle fibers. $9-\mu \mathrm{m}$ thick section. $\times 65$.

Fig. 2 Forty-five-day-old regenerate. Section was incubated to show AChE (MEP) according to Gomori's method. The reaction is localized and typical MEP's (arrows) can be seen. $10-\mu \mathrm{m}$ frozen section. $\times 260$.

Fig. 3 Forty-day-old normal regenerate with SDH reaction. Dark staining fibers are in the lower left corner, while light staining fibers are in the upper right corner. Thus, fiber type differentiation can be seen. Note: Muscle fibers of the same type tend to group together. $10-\mu \mathrm{m}$ frozen section. $\times 65$.

Fig. 4 Forty-day-old normal regenerate with myofibrillar ATPase reaction. Lightly stained fibers (dark arrows) and darkly stained fibers (white arrow) can be seen clearly at this stage. $10-\mu \mathrm{m}$ frozen section. $\times 65$.

Fig. 5 Normal gastrocnemius serial sections showing three fiber types. SDH reaction is on the left and myofibrillar ATPase reaction is on the right. FOG, fast-twitch-oxidative-glycolytic fibers; FG, fasttwitch-glycolytic fibers; SO, slow-twitch-oxidative fibers. $(10-\mu \mathrm{m}$ thick frozen section. $\times 65$.)

Fig. 6 Serial sections of normal regenerate showing three fiber types. SDH reaction is on the left and myofibrillar ATPase reaction is on the right. FOG, fast-twitch-oxidative-glycolytic fibers; FG, fasttwitch-glycolytic fibers; SO, slow-twitch-oxidative fibers. $10 \mu \mathrm{m}$-thick frozen section. $\times 260$. differential activity could be seen in the denervated regenerates, as in normal ones. Muscle fibers with cross striations and peripherally located nuclei could also be seen (fig. 7). The denervated regenerates also established connections with calaneal (Achilles) tendons.

The close similarities between normal and denervated regenerates persisted until about 40 days. Then, the degenerating muscle fibers (fig. 8) in denervated regenerates could be seen more readily than those in early regenerates, whether normal or denervated. In normal regenerates, the muscle fibers became mature and degener-

TABLE 1

Cross-sectional area $\left(\mu \mathrm{m}^{2}\right)$ of muscle fibers in normal and denervated regenerates

\begin{tabular}{ccr}
\hline Age & Denervated & \multicolumn{1}{c}{ Normal } \\
\hline 10 days & $46 \pm 1.35$ & $80 \pm 3.37$ \\
20 days & $88 \pm 2.79$ & $208 \pm 4.19$ \\
30 days & $161 \pm 3.20$ & $299 \pm 8.70$ \\
40 days & $127 \pm 2.64$ & $515 \pm 20.29$ \\
65 days & $84 \pm 2.46$ & $801 \pm 32.01$ \\
90 days & $35 \pm 1.48$ & $1238 \pm 76.02$ \\
150 days & - & $1462 \pm 98.99$ \\
270 days & - & $1523 \pm 103.10$ \\
\hline
\end{tabular}

Samples were collected only from proximal parts of the regenerates. For each scheduled date two animals were used except for days 90,150 , and 270 in which only one animal was used. Each number is a mean \pm S.E.

TABLE 2

Total number of muscle fibers in normal and denervated regenerates

\begin{tabular}{ccc}
\hline Age & Normal & Denervated \\
\hline 10 days & N $1=4763$ & D $1=4870$ \\
& N $2=4148$ & D $2=4572$ \\
20 days & N $1=6921$ & D $1=7848$ \\
& N $2=5961$ & D $2=7608$ \\
30 days & N $1=8709$ & D $1=2653$ \\
& N $2=3993$ & D $2=5118$ \\
40 days & N $1=9018$ & D $1=3456$ \\
& N $2=9333$ & D $2=6876$ \\
65 days & N $1=6451$ & D $1=2623$ \\
& N $2=3476$ & D $2=3231$ \\
\hline
\end{tabular}

Fibers were counted only from the cross sections of the proximal parts of regenerates. $N$, indicates normal regenerates; $D$, indicates denervated regenerates. 1 and 2 , represent first and second animal. 
ating muscle fibers gradually disappeared. In denervated regenerates, degeneration set in gradually. There were two major types of fiber degeneration. The first and most rapid type was fiber swelling with deeply stained sarcoplasm (fig. 8). The nuclei were pycnotic. A second type was sim-
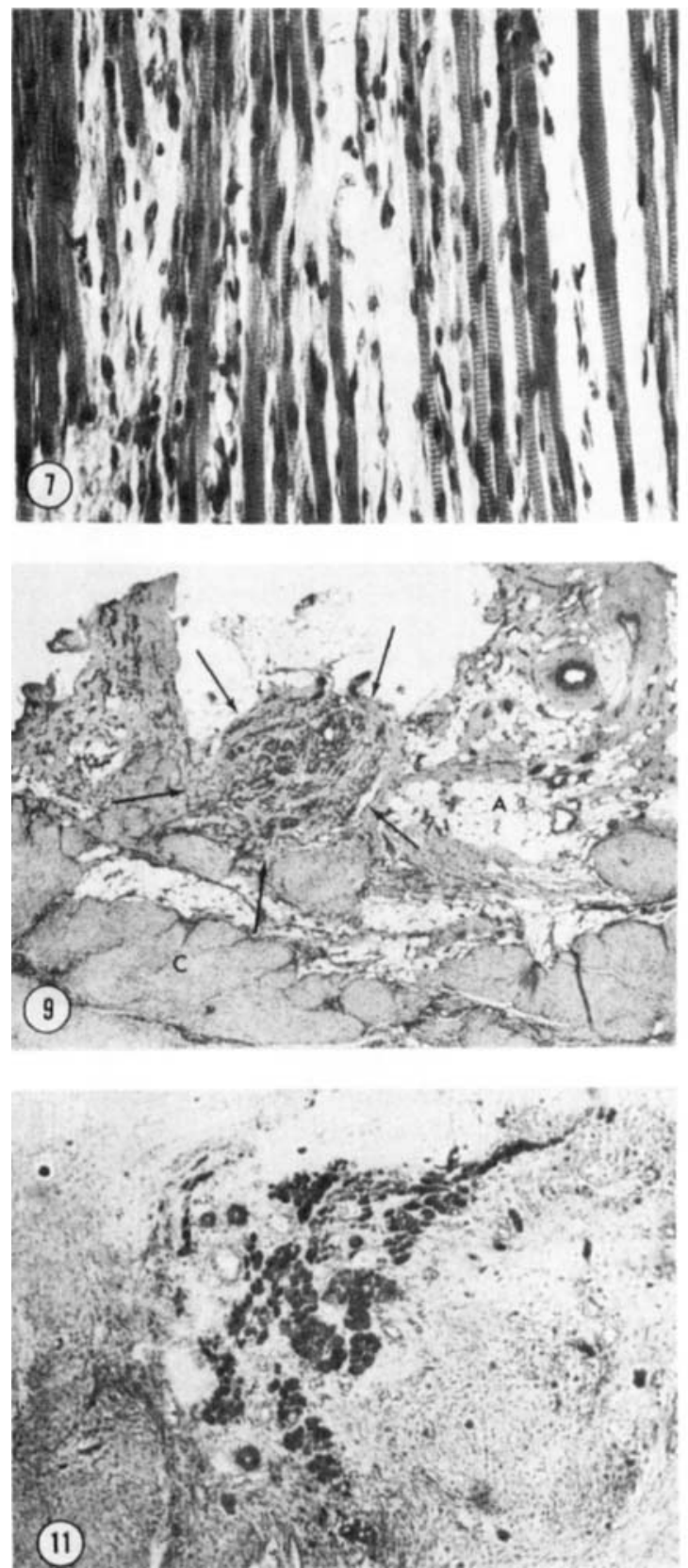

ple reduction in fiber size. No detectable signs of abnormality in sarcoplasm and nuclei were observed. The fibers simply underwent gradual atrophy. Regardless of the type of degeneration, connective tissue and adipose tissue gradually replaced the muscle fibers (figs. 9, 10). The time for
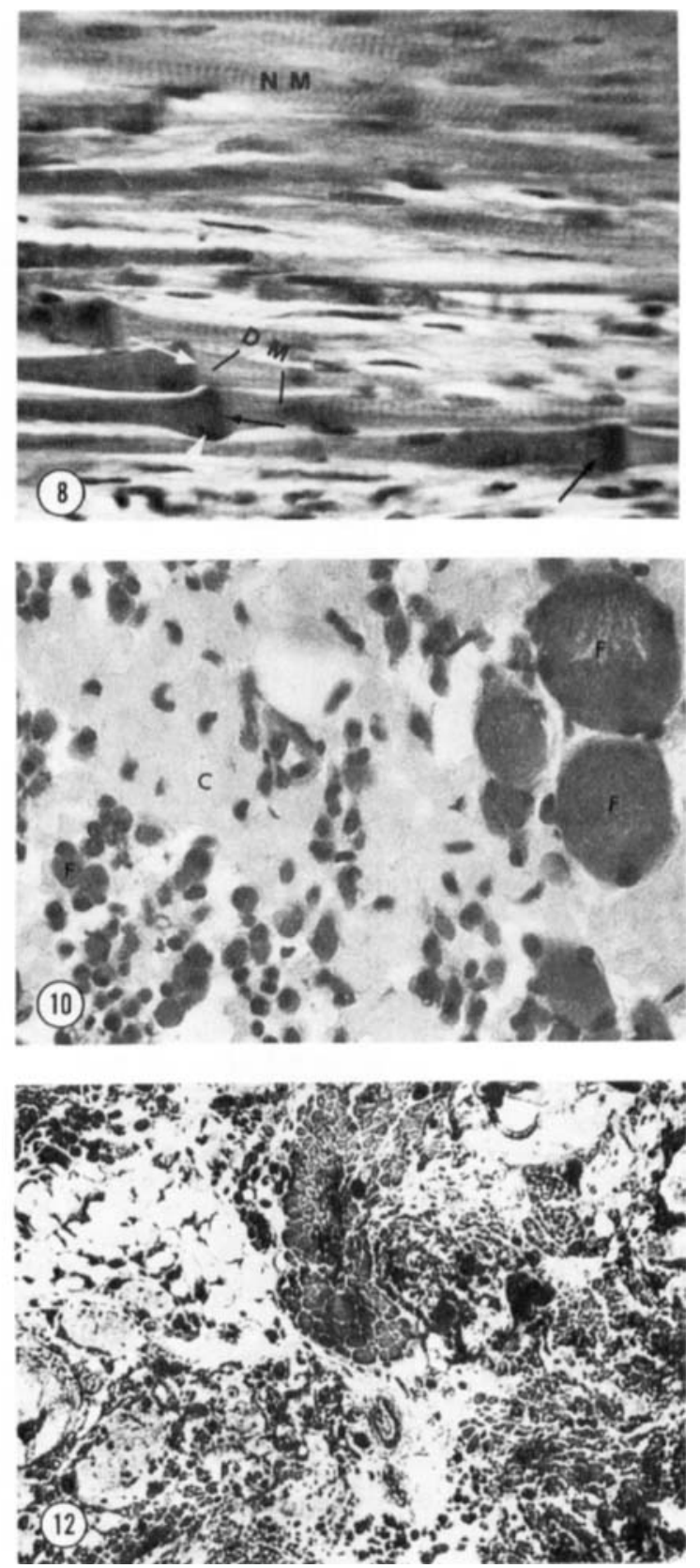
TABLE 3

Number of muscle fibers with peripheral nuclei per 100 fibers counted (P\%, mean $\pm \mathrm{S}$

E.) and number of normal muscle fibers per 50 fibers counted (NM/50, mean $\pm \mathrm{S}$. E.) of normal and denervated regenerates from 10 to 40 days postoperatively

\begin{tabular}{lccccc}
\hline & \multicolumn{2}{c}{$\mathrm{P} \%$} & \multicolumn{2}{c}{ NM/50 } \\
\cline { 2 - 5 } & Normal & Denervated & & Normal & Denervated \\
\hline 10 days & $75.17 \pm 2.24$ & $74.50 \pm 1.45$ & & $36.08 \pm 2.52$ & $36.17 \pm 1.58$ \\
20 days & $90.92 \pm 0.96$ & $85.17 \pm 0.84$ & & $47.17 \pm 0.74$ & $46.17 \pm 1.13$ \\
30 days & $92.08 \pm 0.67$ & $88.92 \pm 1.22$ & & $47.83 \pm 0.44$ & $45.17 \pm 0.65$ \\
40 days & $97.42 \pm 0.51$ & $81.25 \pm 1.29$ & & $50.00 \pm 0.00$ & $25.50 \pm 2.99$ \\
\hline
\end{tabular}

In $\mathrm{P} \%$ of the left column, each number is a percentage and in NM/50 of the right column, each number is a ratio out of 50. Data are only from proximal parts of regenerates and are shown in figures 15 and 16.

complete disappearance of muscle fibers varied with individual regenerates. In some cases, it occurred around 90 days and, in others, traces of fibers could still be seen as late as $\mathbf{1 5 0}$ days postoperatively.

The course of histochemical fiber type differentiation in the denervated regenerates was almost identical to that of normal ones in the early stages (10-30 days). Both SDH and myofibrillar ATPase reactions were positive. By 35 to 40 days, denervated regenerates remained in an undiffer-

Fig. 7 Longitudinal sections of 25 day-old denervated regenerate. Cross striations and peripherally located nuclei can be seen clearly. $1-\mu \mathrm{m}$ thick paraffin section, $\mathrm{H} \& \mathrm{E}$ staining. $\times 65$.

Fig. 8 Degenerating muscle fibers (DM) of a 25day denervated regenerate. Their sarcoplasms are deeply stained and swollen (white arrow); occasionally a retraction cap and clot (dark arrow) can be seen. The nuclei are usually shrunken and pyknotic (white arrow-head). NM, normal muscle fibers. 9- $\mu \mathrm{m}$ thick section, $H \& E$ staining. $\times 65$.

Fig. 9 Cross-section of 90 -day-old denervated regenerate, low power view $(\times 7)$. Connective tissue (C) and adipose tissue (A) become the main constituents of the regenerate. In the central area (encircled by arrows), however, a number of small degenerating fibers can still be seen. $9-\mu \mathrm{m}$ paraffin section, H\&E staining.

Fig. 10 Cross section of 90-day-old denervated regenerate, high power view $(\times 265)$ showing the degenerating muscle fibers in the central area of figure 9 . F, muscle fibers; $\mathrm{C}$, connective tissue. $9-\mu \mathrm{m}$ paraffin section, H\&E staining.

Fig. 11 Forty-day-old denervated regenerate with SDH reaction. No indication of fiber type differentiation can be seen. The reaction remains positive. $10-\mu \mathrm{m}$ frozen section. $\times 65$

Fig. 12 Forty-day-old denervated regenerate with myofibrillar ATPase. The reaction remains positive, but fiber type differentiation has not occurred. $10-\mu \mathrm{m}$ frozen section. $\times 42$. entiated primitive stage (figs. 11, 12), while normal ones had begun to differentiate (figs. 3,4 ). As time went on, the enzymatic activity of denervated regenerating fibers became progressively weaker. There was no indication throughout the study of heterogeneous fiber type differentiation.

\section{Series III: Quantitative comparisons between normal and denervated regeneration}

(1) The mean cross sectional areas of regenerating muscle fibers are shown in table 1 and figure 13. They show that muscle fibers increased in cross sectional areas in normal and, in the early stages, also in the denervated regenerates. The major increase in the normal regenerates took place between 30 and 150 days. In the denervated regenerates, the mean cross sectional areas increased to a maximum at 30 days and then decreased gradually. The frequency distribution of different muscle fiber cross sectional areas is shown in figure 14. It shows that the percentages of different fibers cross sectional area are comparable between normal and denervated regenerates up to 30 days. After 30 days, most fibers in normal regenerates moved to large fiber range. On the contrary, most of the muscle fibers in denervated regenerates became reduced in cross sectional areas and fell into small fiber range.

(2) Table 2 shows the number of muscle fibers in normal and denervated regenerates. It indicates:

(a) The number of muscle fibers varied considerably among the regener- 


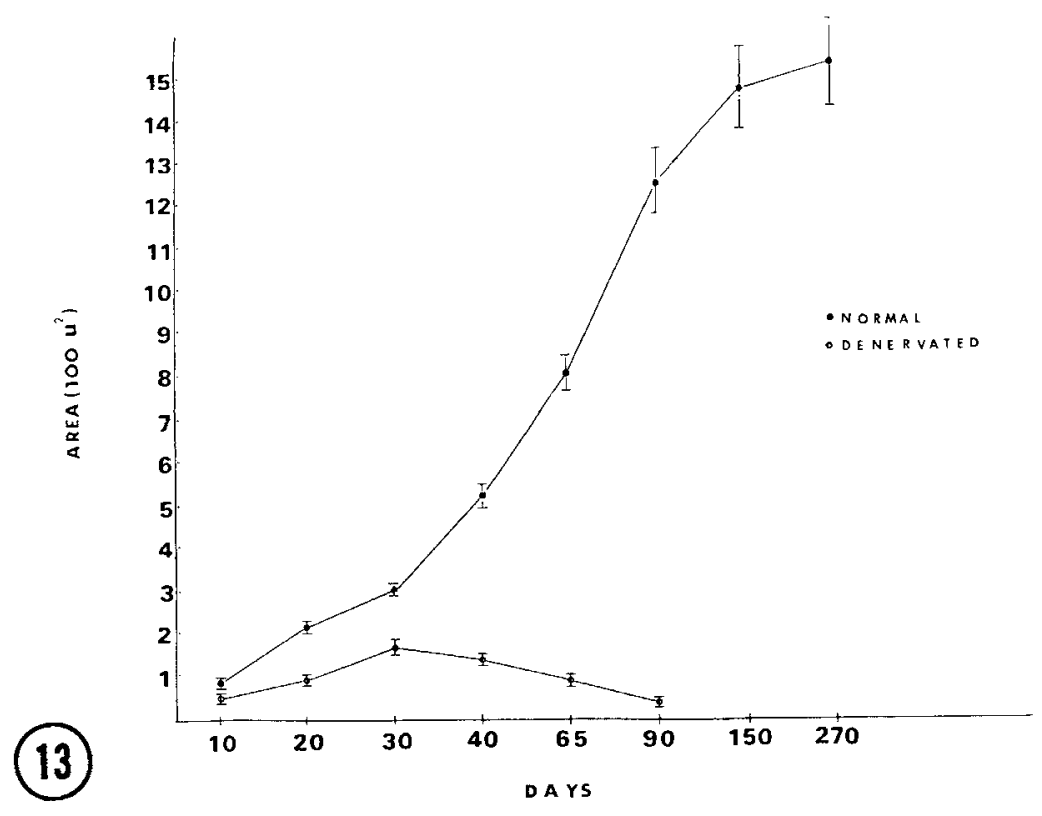

Fig. 13 Mean cross-sectional area of normal and denervated regenerating muscle fibers.

ates. This occurred not only among regenerates of different age groups but also among regenerates in the same age group.

(b) Substantial numbers of muscle fibers persisted even in regenerates deprived of nerves for 65 days.

(c) In early regenerates, the numbers of fibers in normal and denervated regenerates were similar to one another.

(3) Figure 15 and table 3 show the percentage of muscle fibers with peripheral nuclei per 100 nucleated fibers counted (P\%). Figure 16 and table 3 show the percentage of normal muscle fibers (as opposed to degenerating muscle fibers) per 50 fibers counted (NM/50). It indicates that there was a parallelism between normal and denervated regenerates in terms of $\mathrm{P} \%$ and NM/50 and no statistical differences exist up to 30 days. At 40 days, however, significant statistical differences between normal and denervated regenerates become evident.

\section{DISCUSSION}

The results of the present investigation show that MEP's and three fiber types will return to the normal innervated regener- ates, and the events of their differentiation resemble those of embryonic myogenesis (Tennyson et al., '73; Dubowitz, '65a,b). Most muscle fibers in the normal regenerates showed positive myofibrillar ATPase reaction and are thus classified as fast fibers. Guth ('73) cautioned that positive ATPase reaction might not necessarily represent fast fibers. Nevertheless, the present investigator feels that these fibers are fast type because contractile studies (Carlson and Gutmann, '72) revealed that the regenerates behave like fast contracting muscles.

The intrinsic ability of myogenic cells to develop in the absence of nerves can be clearly demonstrated in the early phases of denervated muscle regeneration. The evidence is as follows: (1) After approximately 15 days, no myotubes could be found in either case and all myogenic tissues had developed to the stage of early muscle fibers, indicating that the rates of development were similar. (2) Up to 30 days, denervated and normal regenerates were comparable with regard to percentage of muscle fibers with peripheral nuclei and the percentage of normal muscle fibers per 50 fibers 


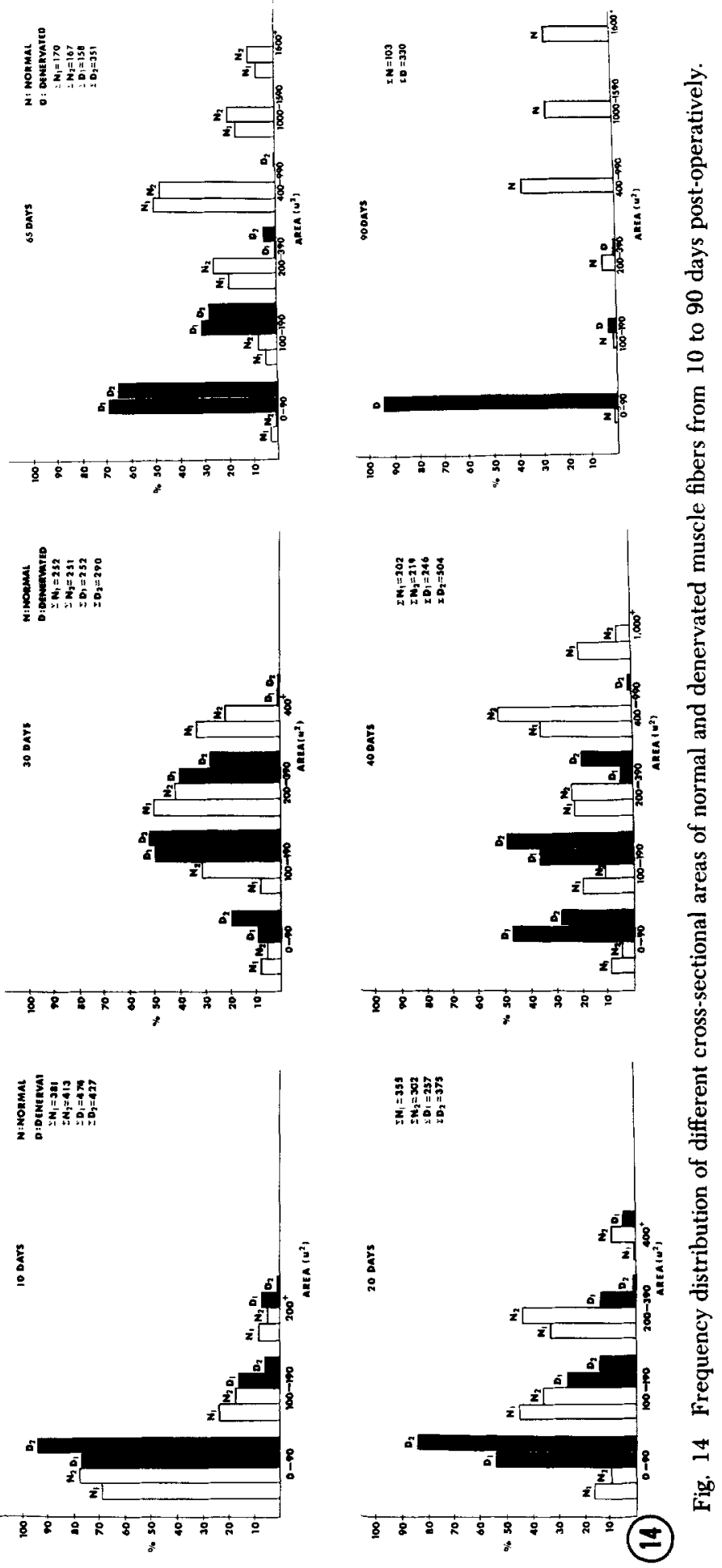




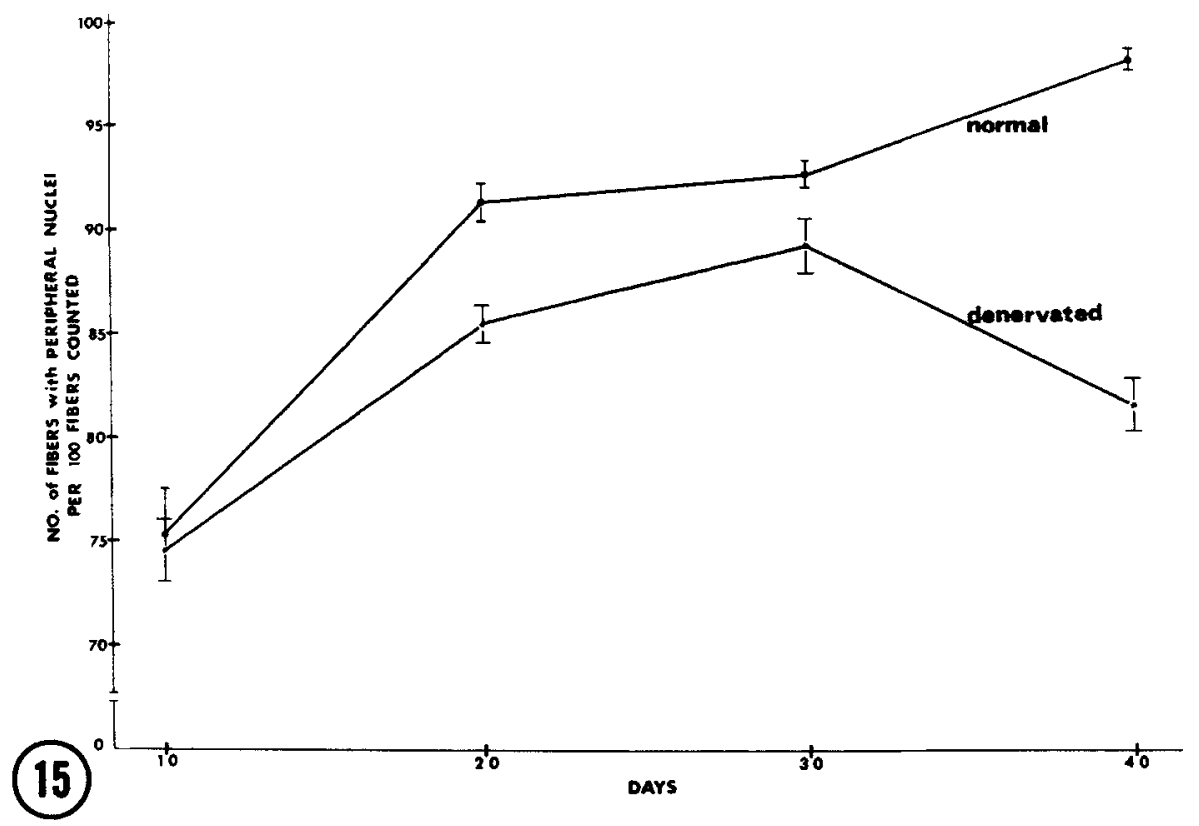

Fig. 15 Number of muscle fibers with peripheral nuclei per 100 fibers counted of normal and denervated regenerates. Data are taken from the proximal part of the regenerates only. No significant differences exist from 10 to 30 days between normal and denervated regenerates. At 40 days, however, the difference becomes apparent.

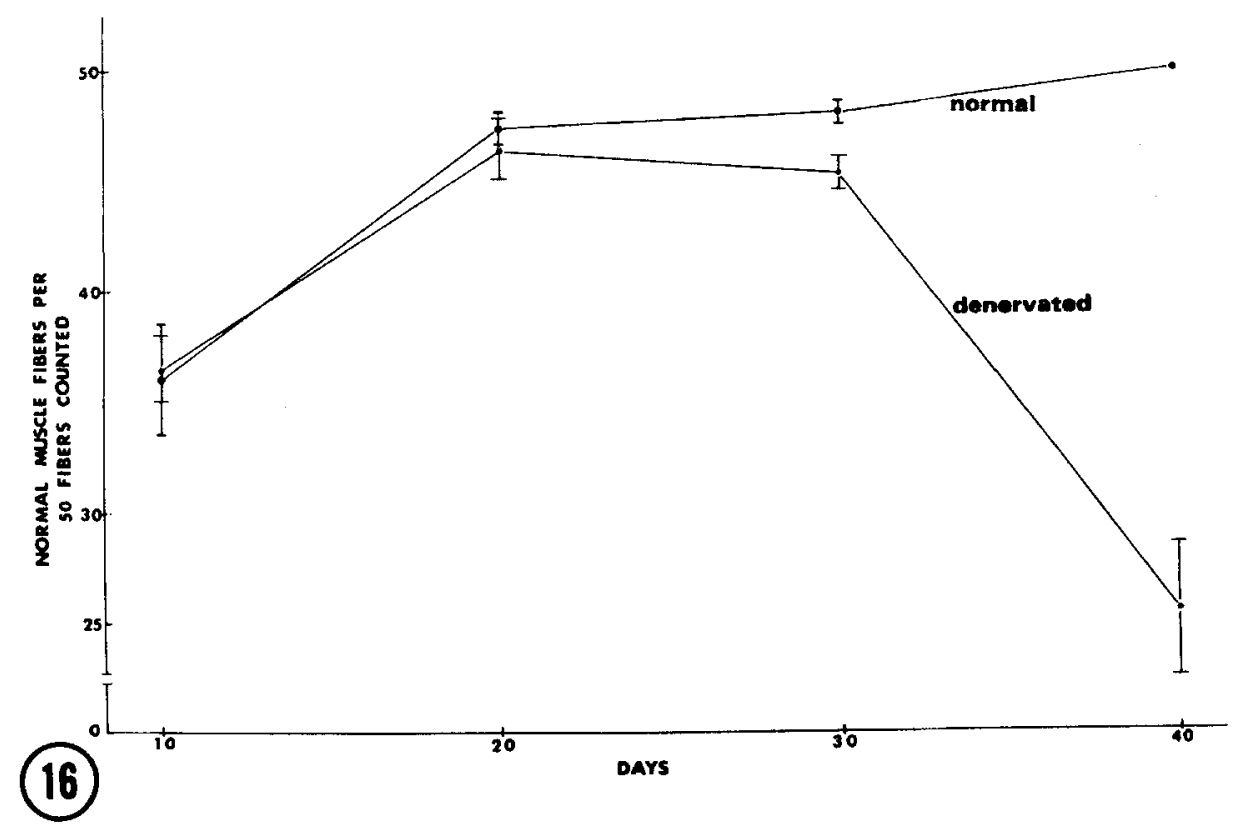

Fig. 16 Number of normal muscle fibers per 50 fibers counted of normal and denervated regenerates. Data are taken from proximal part of the regenerates only. No significant differences exist from 10 to 30 days between normal and denervated regenerates. On 40 days, however, the difference becomes apparent. 
counted. (3) The two types of regenerates were also comparable in the total numbers of muscle fibers. (4) The regenerated muscle fibers in both cases showed clear cross striations. (5) Both SDH and myofibrillar ATPase reactions were positive and primitive in denervated regenerates and they could not be distinguished from those of normal ones up to 30 days postoperatively. This intrinsic ability of myogenic tissues to differentiate has also been observed in other regeneration studies (Allbrook and Aitkin, '51; Hall-Craggs and Seyan, '75), and in the studies on muscle ontogeny (Zelena, '62; Engel and Karpati, '68; Shafiq et al., '72).

The only consistent difference between normal and denervated regenerates in the early stages was the smaller fiber cross sectional areas in the denervated regenerates. First, the small cross sectional area might not necessarily imply abnormality. Second, it could well be due to the lack of use of the denervated legs rather than lack of nerve fibers, since it is well known that use and disuse of muscle affects muscle fiber diameters (Edgerton et al., '72; Maier et al., '73; Tomanek and Lund, '74). In the case of denervated regenerates, the leg was permantly flexed and not used. Thus the small fiber cross sectional area could be due to the lack of exercise.

Starting around $\mathbf{4 0}$ days, differences between normal and denervated regenerates became evident. Histochemically, the normal regenerates were in the last stage of development-histochemical fiber type differentiation. In the denervated regenerates, however, there were no indications of such activity, although both SDH and myofibrillar ATPase remained positive for a long time. Morphologically, degenerating muscle fibers could be easily seen. Thus, nerves seem to be important in the late phase of regeneration, namely the differentiation of fiber types and maintenance of structural integrity of muscle fibers. This view is also shared by Hall-Craggs and Seyan ('75) and Hsu ('71) in regeneration studies, Shafiq et al., ('72) and Engel and Karpati ('68) in ontogenic studies and
Askanas et al. ('72) and Crain ('70) in tissue culture studies.

Contractile properties of the denervated regenerates were not studied in the present investigation. Carlson and Gutmann (72) observed contractile activities of innervated regenerates upon direct stimulation as early as eight days postoperatively. At this early date, functional contact between nerves and muscle fibers has not developed yet. In recent studies with denervated sliced muscle transplantation experiments, Carlson and Gutmann (personal communication) also observed contractile activity in the denervated transplants. Thus, one would speculate that the denervated minced muscle regenerates would also be contractile upon direct stimulation.

\section{ACKNOWLEDGMENT}

The author would like to thank Dr. Bruce M. Carlson for his patience and careful guidance in carrying out this research and Mr. Peter P. S. Ruey for his assistance in statistical analysis.

\section{LITERATURE CITED}

Allbrook, D. B., and J. T. Aitkin 1951 Reinnervation of skeletal muscle after acute ischemia. J. Anat. (London), 85: 376-390.

Askanas, V., S. A. Shafiq and A. T. Milhorat 1972 Histochemistry of cultured aneural chick muscle: morphological maturation without differentiation of fiber types. Exp. Neurol., 37: 218-230.

Carlson, B. M. 1968 Regeneration of the completely excised gastrocnemius muscle in the frog and rat from minced muscle fragments. J. Morph., $125: 447$. 471 .

1970 The regeneration of entire muscle from minced fragments. In: Regeneration of Striated Muscle and Myogenesis. A. Mauro, S. A. Shafiq and A. T. Milhorat, eds. Excerpta Medica, Amsterdam, pp. 194-211.

Carlson, M. B., and E. Gutmann 1972 Development of contractile properties of minced muscle regenerates in the rat. Exp. Neurol., 36: 239-249.

Crain, S. M. 1970 Bioelectric interactions between cultured fetal rodent spinal cord and skeletal muscle after innervation in vitro. J. Exp. Zool., 173: 353-370.

Dubowitz, V. 1965a Enzyme histochemistry of skeletal muscle, Part I (developing animal muscle). J. Neurol. Neurosurg. Psychiat., 28: 516-519.

$1965 \mathrm{~b}$ Enzyme histochemistry of skeletal muscle, Part II (developing human muscle). J. Neurol. Neurosurg. Psychiat., 28: 519-524. 
Edgerton, V. R., R. J. Barnard, J. B. Peter, C. A. Gillespie and D. R. Simpson 1972 Overloaded skeletal muscles of a nonhuman primate (Galago senegalinsis). Exp. Neurol., 37: 322-339.

Engel, W. K., and G. Karpati 1968 Impaired skeletal muscle maturation following neonatal neurectomy. Devel. Biol., 17: 713-723.

Guth, L. 1973 Fact and artifact in histochemical procedure for myofibrillar ATPase. Exp. Neurol,, 41: 440-450.

Hall-Craggs, E. C. B., and H. S. Seyan 1975 Histochemical changes in innervated and denervated skeletal muscle fibers following treatment with Bupivacaine (Marcain). Exp. Neurol., 46: 345-354.

Hsu, L. 1971 The role of nerves in the regeneration of minced skeletal muscle in adult anurans. Ph.D. Diss., Univ. of Michigan.

Hudgson, P., and E. J. Field 1973 Regeneration of muscle. In: The Structure and Function of Muscle. Vol. II. G. H. Bourne, ed. Academic Press Inc., New York, pp. 312-363.

Maier, A., E. Eldred and V. R. Edgerton 1973 The effects on spindles of muscle atrophy and hypertrophy. Exp. Neurol., 37; 100-123.

Mong, F. S. F. 1975 Nervous influence on minced muscle regeneration in rats. Anat. Rec., 181: 429.

Nachlas, M. M., K. C. Tsou, E. de Souza, C. S. Cheng and A.M. Seligman 1957 Cytochemical demonstration of succinic dehydrogenase by the use of new p-nitrophenyl substituted ditetrazole. J. Histochem. and Cytochem., 5: 420-436.

Padykula, H. A., and E. Herman 1955 Factors affecting the activity of adenosine triphosphatase and other phosphatases as measured by histochemi- cal techniques. J. Histochem. and Cytochem., 3: 161-169.

Pearse, A. G. E. 1972 Histochemistry. Vol 2. Third ed. Churchill Livingstone, London.

Peter, J., R. J. Barnard, V. R. Edgerton, C. Y. Gillespie and K. E. Stempel 1972 Metabolic profiles of three fiber types of skeletal muscle in guinea pigs and rabbits. Biochemistry, 11: 2627-2633.

Shafiq, S. A., S. A. Asiedu and A. T. Milhorat 1972 Effect of neonatal neurectomy on differentiation of fiber types in rat skeletal muscles. Exp. Neurol., 35: 529-540.

Studitsky, A. N., R. P. Zhenevskaya and O. N. Rumyantseva 1963 The role of neurotrophic influences upon the restitution of structures and function of regenerating muscles. In: The Effect of Use and Disuse in Neuromuscular Functions. E. Gutmann and P. Hnik, eds. Publ. House. Czechoslovak Acad. Sci., Prague, pp. 71-81.

Tennyson, V. M., M. Brzin and L. Kremzner 1973 Acetylcholinesterase activity in the myotube and muscle satellite cell of the fetal rabbit. J. Histochem. Cytochem., 21: 634-652.

Tomanek, R. J., and R. D. Lund 1974 Degeneration of different types of skeletal muscle fibers. II. Immobilization. J. Anat., 118: 531-541.

Winer, B. J. 1971 Statistical Principle in Experimental Design. Second ed. McGraw Hill Co., New York.

Zelena, J. 1962 The effect of denervation on muscle development. In: The Denervated Muscle. E. Gutmann, ed. Publishing House of Czech. Acad. Sci., Prague, pp. 103-126.

Zhenevskaya, R. P. 1962 Experimental histologic investigation of skeletal muscle tissue. Rev. Canad. Biol., 21: 457-470. 